\title{
Design and Construction of Gas Fire Alert System
}

\author{
Lorinda E. PASCUAL ${ }^{1}$, Noel T. FLORENCONDIA ${ }^{2}$, Christopher M. LADIGNON ${ }^{3}$, Erbert Alison J. \\ BENEDICTO $^{4}$, Jeffrey C. CUDAPAS ${ }^{5}$, Sam Carlo R. LADORES ${ }^{6}$
}

1,2,3,4,5,6 Nueva Ecija University of Science and Technology Gen. Tinio Street

Cabanatuan City 3100

\begin{abstract}
This research paper aspired to design and develop a gas leakage and fire monitoring and detection alarm system based on SMS. Most fire incidents in houses, schools and factories are caused by gas leakage. Many houses and factories are being destroyed by fire and gas leakage. To prevent the loss of life and properties, preventive measures should be taken, hence this research was conceptualized. The installation of a GF (Gas and Fire) alert system to vulnerable locations may help lessen if not totally eliminate the dangers posed by gas leakage and fire.

The GF system was designed and develop to prevent the loss of life and properties caused by gas leakages \& fire thus ensuring the safety of life and property. The design of the GF system is composed of hardware and software integrated and programmed to work as gas and fire detector. The programs are coded using embedded C-language. Tests performed on the developed GF system shows excellent response in detecting gas leaks and fire with short time delay. On the other hand, sending of SMS alert shows promising results with few seconds time delay. Also, the network signal of the place of installation affects the performance of the device to send text messages.
\end{abstract}

KEYWORDS: Gas, Fire, GSM, Alarm System

\section{INTRODUCTION}

Liquefied Petroleum Gas which is usually referred to as LPG was first introduced in 1910 by Dr. Walter Snelling. LPG is a mixture of highly flammable chemicals namely commercial propane and commercial butane containing saturated as well as unsaturated hydrocarbons. Since LPG is an odorless gas, leakage cannot be easily detected. To give odor for easy detection of the gas, ethane oil is added so that leakage of this gas can be easily detected. Over the years since it was first introduced, the demand for LPG continues to rise because of its many uses. LPG can now be used in homes not only for cooking purposes but also for central heating, hot water, mobile heaters for outdoor activities such as boats, caravans and barbecues and others. Although the use of LPG in homes is a very convenient way of producing energy, there is a threat posed concerning its leakage. The LPG is heavier than air, and when inhaled, it does not disperse easily and can lead to suffocation. The leaked LPG can also lead to an explosion when ignited.

One cannot deny the importance and usefulness of fire in our daily life. It provided us the first form of portable light and heat. It also gave us the ability to cook food, to forge metal instruments, to make pottery, to make hard bricks, and to fuel electric generating plants among others. We cannot imagine a world without fire. It is definitely regarded as one of the most powerful discovery in the history of mankind. And yet, although it is very useful and can be equally dangerous. At an instant it can kill people and destroy our houses and all our belongings. Fire hazard in homes and buildings have become a growing concern all over the globe in recent years. In the past two decades (1993-2015), a total of 86.4 million fire incidents have caused more than one million fire deaths [1]. Fire can also turn a whole forest into a rubbish and ash. Forest fire is considered as the most common hazard in forests. Compared to other forces of nature such as earthquakes, floods and other calamities, fire claims the life of more individuals each year.

One of the most traumatic experience a family can suffer is the trauma brought by a burning house. Almost half of all fires occurring in homes are caused by cooking according to the Federal Emergency Management Agency (FEMA) [2]. Compared to other rooms in the house like the living room, bedrooms or bathrooms, most house fires start in the kitchen. One of the reasons can be attributed to leaked LPG. The fire per se. and fire due to leakage of dangerous and flammable gases like LPG may result to destructive house fire. This can be considered as a major concern in household, commercial premises and other areas where LPG is handled and used. Gas leakage and fire may not only lead to financial loss. Due to loss or damage to property but most importantly the loss of human lives. The good news is that, this type of accident can be prevented. You can protect yourself and your love ones of experiencing the tragedy brought upon by fire 
simply by knowing where and when fires are most likely to start.

This paper provides an effective solution for gas leakage and fire detection thus preventing the loss of life and property. The developed GF system when installed in areas vulnerable to gas leakage and fire audibly alerts the users when these occur. The GF system also informs an authorized person of the detected gas leakage and fire by sending him a message through SMS. The GF's system such as microcontroller, sensor and software are designed programmed and built and integrated to function as gas leakage and fire detector. As such, the developed GF system is designed and constructed to prevent house fires due to gas leakage and thus also preventing the loss of life and property.

\section{OBJECTIVES}

The research aimed at designing, constructing and testing the performance of the Gas-Fire (GF) Alert trough SMS. Specifically, the research has the following specific objectives.

1. To design and construct GF alert system.

2. To determine the time delay of gas leakage and fire sensor in sending SMS alert

3. To determine the effect of the place of installation of the device.

\section{METHODOLOGY}

\section{Research Design}

In realizing the objectives of the research, the researchers used the experimental research design. This design was utilized in order to develop an efficient and functional GF system based on the result of the testing on the variables in the study.

\section{Materials}

The materials used in the design and construction of the GF system proposed in this research paper are determined through literature search and integrated the use of affordable instruments, compatibility and wireless communication.

The following are the materials for the construction of GF Alert:
1. 2-Arduino Uno R3
2. SIM 900A Mini GSM Module
3. MQ-2 Gas Sensor Module
4. Fire Sensor Module
5. $16 \times 2$ LCD Module (JHD162A)

6. $5 \mathrm{~V}$ Buzzer

7. Bread board and PCB

8. 2-9V, 1000mA DC Power Supply Unit

9. Connecting wires

10. NPN Transistor (2N2222)

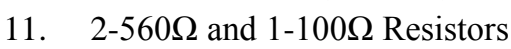

12. $10 \mathrm{k} \Omega$ Potentiometer

13. Green LED

14. 2-way Toggle Switch

15. One whole double sided white board

16. One whole corrugated plastic

17. Wood glue

\section{Procedures of the Study}

The flowchart represents the step by step procedure of the study to be conducted. The corresponding functions of the blocks are the following:

Circuit Designing and Planning - The researchers collected the primary information required to construct the system by doing desktop researches.

Construction - The researchers assembled the prototype of the system. It is an alert system driven by a gas and fire sensors.

Trial Run - The device undergoes a trial run to see any margin of error before the researchers test it under different circumstances. If the device works according to the expected function, the researchers will move to the next step. Otherwise, the researchers will reassemble the device to achieve the minimal margin of error.

Testing of the device - The device was tested in a closed environment and will be observed under different conditions. The functionality of the device was tested based on time delay of gas leakage and fire sensor in sending SMS and the effect of the place of installation of the device.

Evaluation of the device - The device was assessed according to its performance during the testing. The gathered data and information under different conditions and variables were considered in evaluating the device.

Conclusion and Recommendation - After recording, analyzing and interpreting the gathered data and information, conclusion and recommendations were drawn out for future studies.

The procedures of the study are shown in Figure 1. 
Figure 1. Procedures of the Study

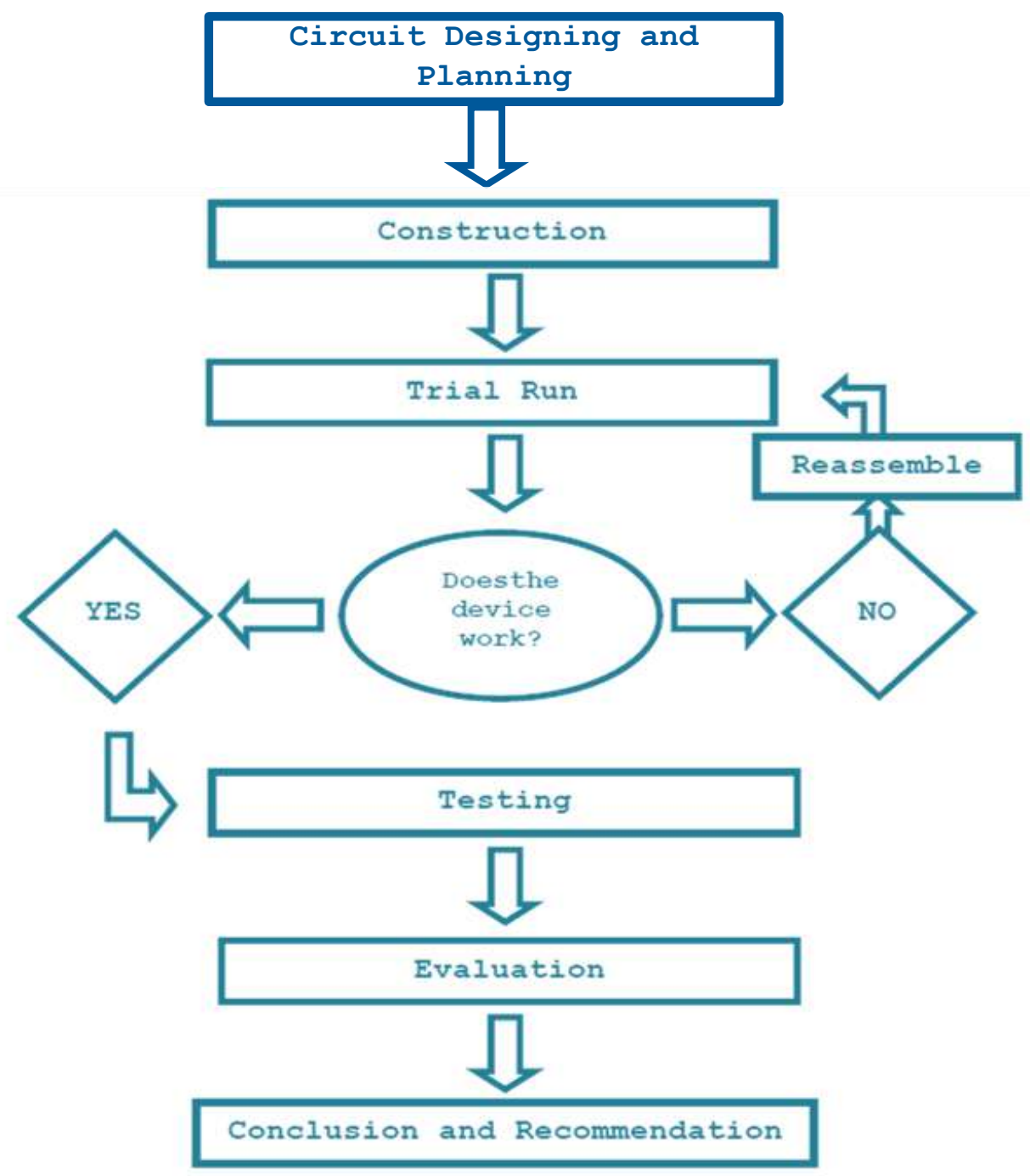

\section{RESULTS AND DISCUSSIONS}

The results of the analyses in the order of the specific objective is presented below.

1. Designing of the GF Alert System

1.1. Block Diagram

Figure 2 Block Diagram of GF Alert

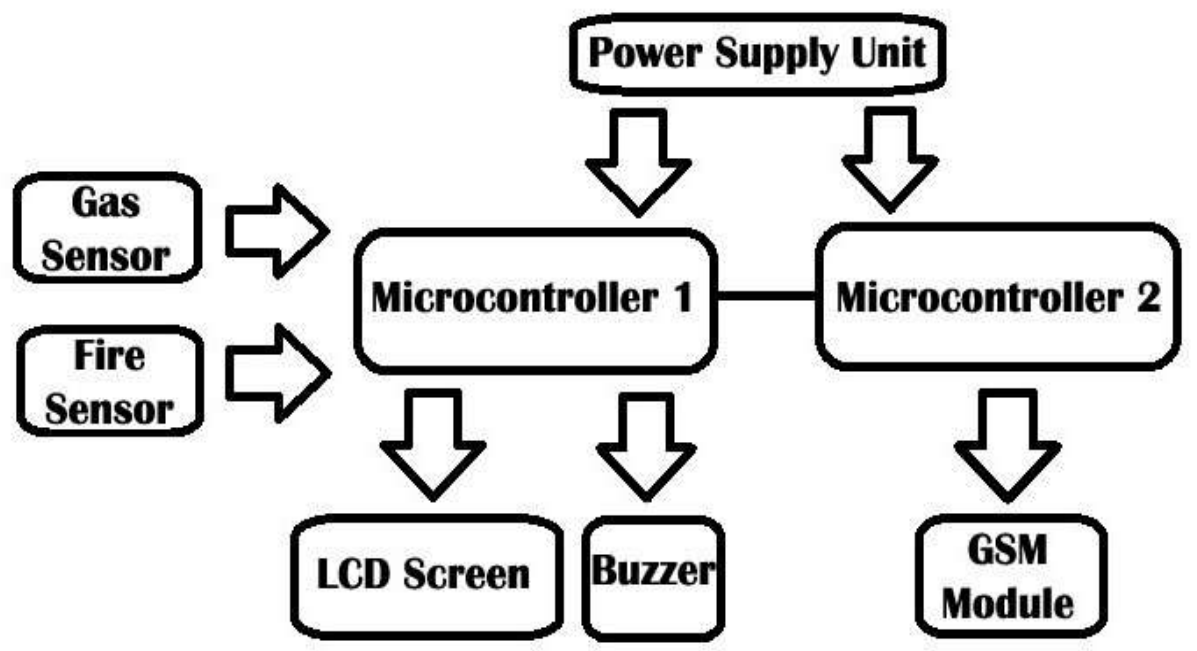


Figure 2 shows the block diagram compose of the different parts of the GF system. The functions of the blocks indicated in the diagram are the following:

Power Supply Unit - This will convert the $230 \mathrm{~V}$ available main voltage to $9 \mathrm{~V}$ DC which is used to power the system.

Microcontroller - It serves as the central point of the GF system. . It is an Arduino UNO R3, a microcontroller board. It is composed of 14 digital input/output pins, 6 analog inputs, an on-board resonator, a reset button, and holes for mounting pin headers. A six-pin header can be connected to an FTDI cable breakout board to provide USB power and communication to the board. It will be used to control the LCD Screen and Buzzer when gas and fire are detected. The input/ output ports of the microcontroller are used for this purpose.

Gas Sensor (MQ-2) - It is a helpful detector for gas leakage. It is ideal for H2, LPG, CH4, CO, and alcohol detection. Because of its high sensitivity and fast reaction time, it is possible to take measurements as soon as possible. By using the potentiometer, the sensitivity of the sensor can be changed. The output of this sensor is 'high' under normal conditions, and when the LPG is sensed, it goes 'low'. [3]
Fire Sensor - This module can easily detect both fire and radiation. It can also detect a natural light source in the $760 \mathrm{~nm}-1100 \mathrm{~nm}$ wavelength range. The distance for detection is up to $100 \mathrm{~cm}$. The module can give a digital output or an analog signal through the Flame sensor.

Buzzer - This is a signaling device in an audio form.

LCD Module - The electronic display module is the LCD (Liquid Crystal Display) screen. The LCD screens are inexpensive, can be easily programmed and have no limitations in displaying special \& even custom characters. It will indicate the status of the operation of the system as: Device is Scanning, Gas detected, fire Detected.

GSM Module - A mobile communication modem is a Global System for Mobile Communication (GSM) modem that can be either a mobile phone or a modem device that can be used to communicate over a network with a computer or some other processor. It will be the responsibility of this unit to send users SMS warnings. [4]

\subsection{Circuit Diagram}

The circuit diagram of the GF system is shown in Figure 3.

Figure 3. Circuit Diagram of GF Alert

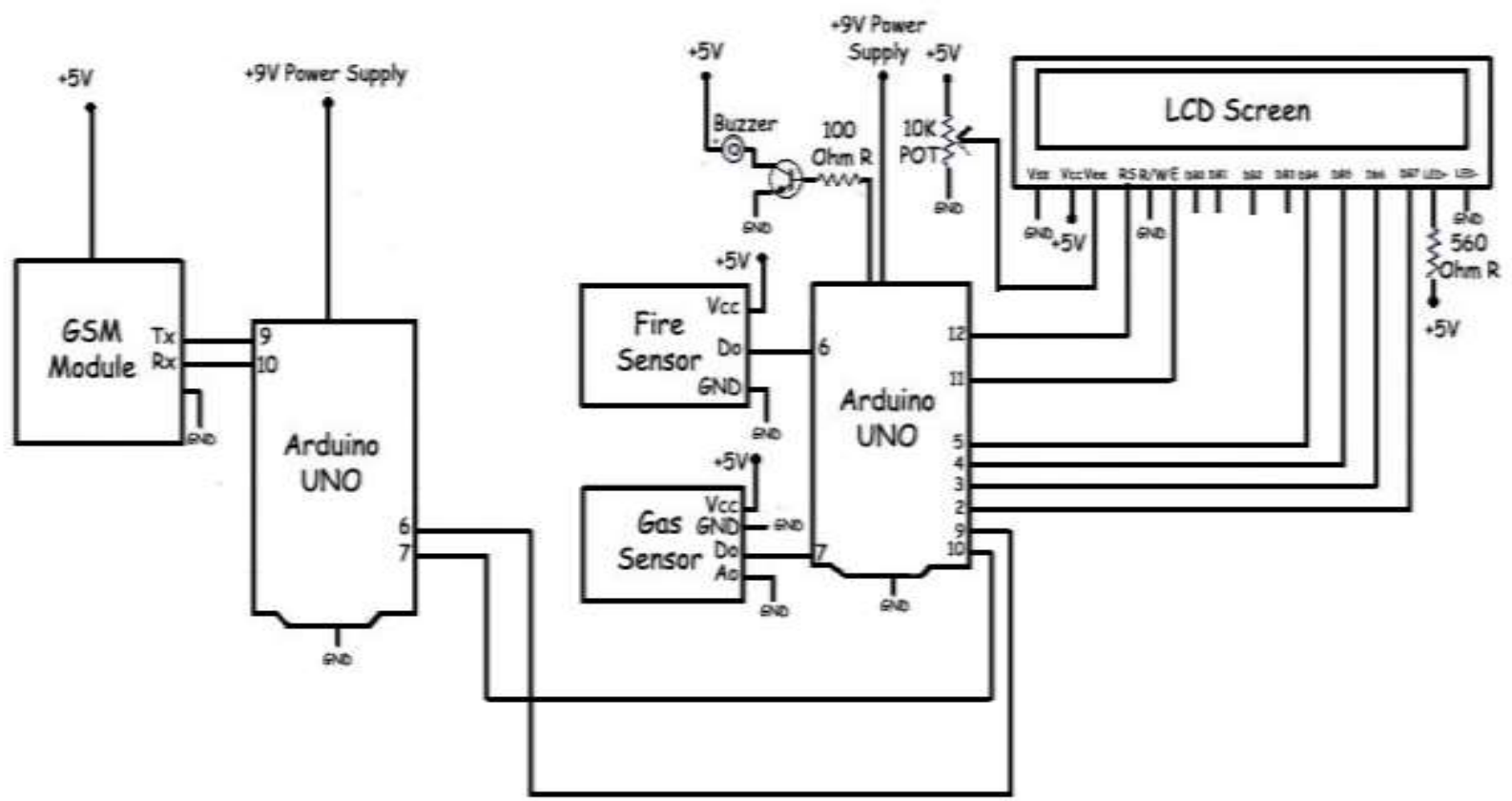

The circuit diagram shows the electrical connection of the materials. Connected on the first Arduino UNO are LCD Screen, Fire Sensor module, Gas Sensor Module and the 5V buzzer while on the second microcontroller, GSM module is connected.

The purpose of having two Arduino UNO in the circuitry is because GSM module needs separate supply. The module must be supplied of $1000 \mathrm{~mA}$ to perform its operation.

\subsection{Construction of the Device}

1.) Using the materials mentioned above, install it on the way just like the circuit diagram.

2.) When it has been connected properly apply the code on the Arduino in order the device to work. 
3.) Test the device before installing. If the system is working, install it in a case.

4.) In construction of the case cut off the double sided white board with the dimension of $21 \mathrm{~cm} \mathrm{x} 15 \mathrm{~cm} \mathrm{x}$ $7 \mathrm{~cm}$

5.) Using wood glue unite the white board cut off in order to build a box where in the top is movable.
6.) Cover the box with corrugated plastic, this will help to prevent water in contact with the integrated circuit.

7.) Create a hole for the LCD, Gas sensor, Flame sensor, and buzzer.

\subsection{The Finished Product}

After the assembly of the materials together with the case the finished product has been made.

Figure 4. Isometric View

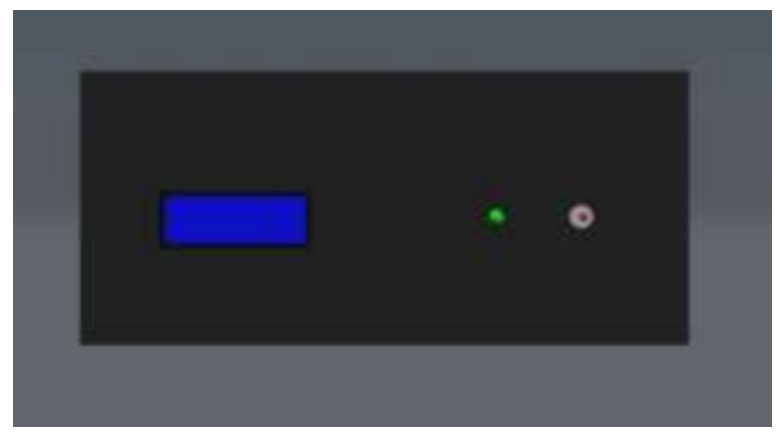

Figure 5. Front View

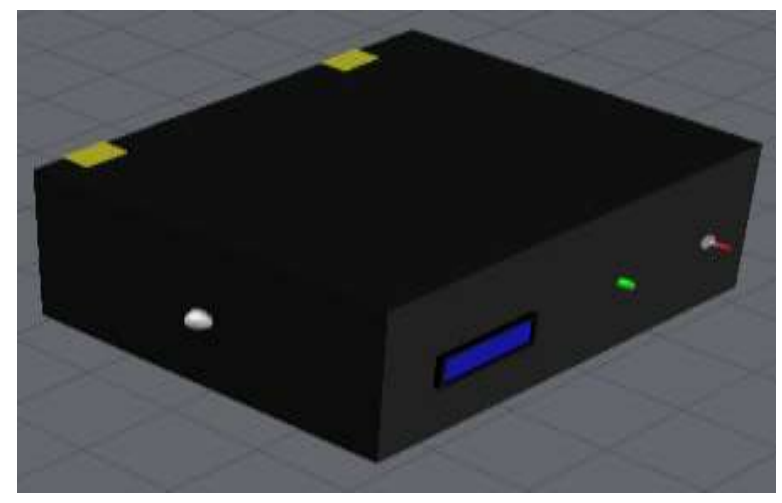

Figure 6. Back View

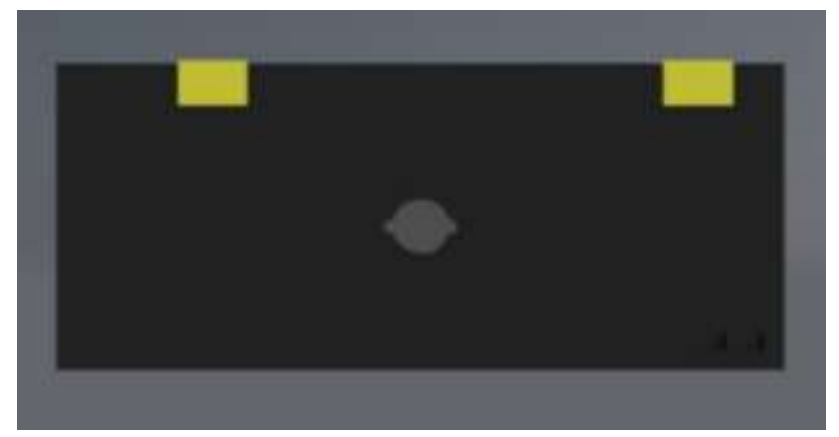

Figure 7. Left Side View

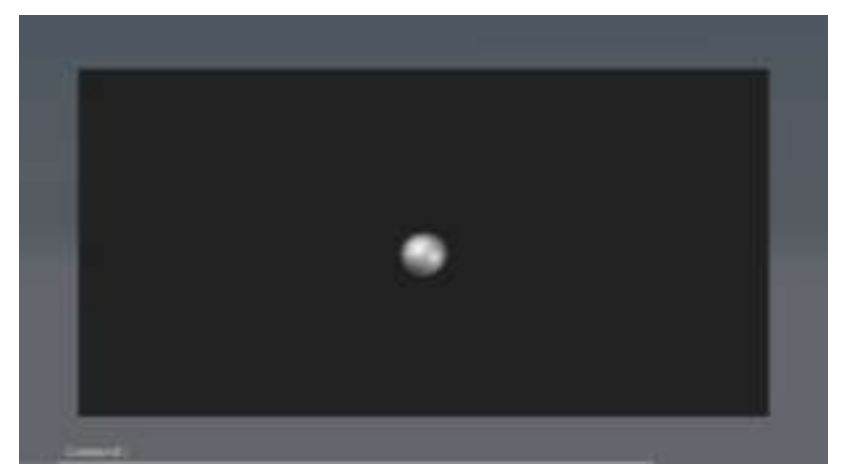


Figure 8. Right Side View

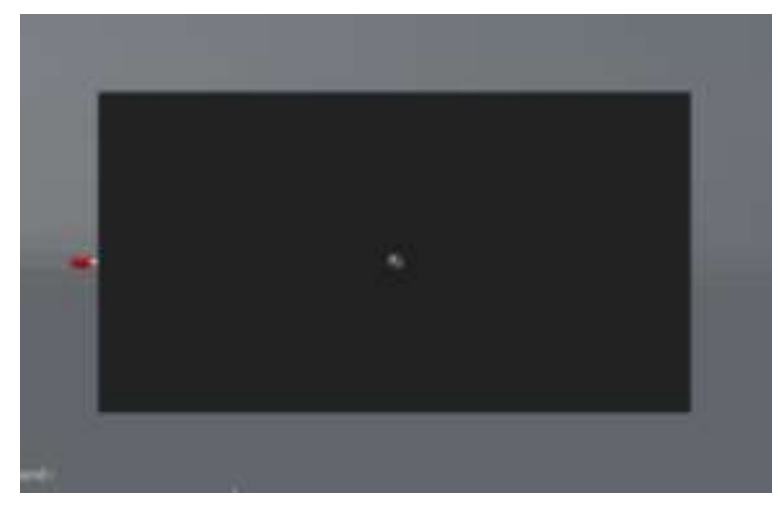

\section{Time Delay of Gas Leakage, Fire Sensor and in Sending SMS Alert}

After the GF Alert has been constructed, the system has been tested to gather data for its functions. The result of the test conducted on the GF system based on the time gas leakage and fire can be detected with respect to location is shown in Tables 1.

Table 1. Testing GF Alert in detecting of Gas and Fire and setting sound alarm

\begin{tabular}{|l|l|l|l|l|l|}
\hline & Test 1 & Test 2 & Test 3 & Test4 & Test 5 \\
\cline { 2 - 6 } & Living Room & Terrace & Bedroom & Dining Area & Kitchen \\
\hline Gas Leakage & $0.75 \mathrm{~s}$ & $1.19 \mathrm{~S}$ & $0.49 \mathrm{~s}$ & $0.63 \mathrm{~s}$ & $0.35 \mathrm{~s}$ \\
\hline Fire & $0.28 \mathrm{~s}$ & $0.97 \mathrm{~s}$ & $0.15 \mathrm{~s}$ & $0.46 \mathrm{~s}$ & $0.22 \mathrm{~s}$ \\
\hline
\end{tabular}

Table 2 shows the time for the GF system to send SMS alert upon detection of fire and gas leakage on various location in the home.

Table 2. Testing of GF Alert in Sending SMS Alert

\begin{tabular}{|l|l|l|l|l|l|}
\hline & Test 1 & Test 2 & Test 3 & Test 4 & Test 5 \\
\cline { 2 - 6 } & Living Room & Terrace & Bedroom & Dining Area & Kitchen \\
\hline Gas Leakage & $8.76 \mathrm{~s}$ & $6.93 \mathrm{~s}$ & $12.09 \mathrm{~s}$ & $10.15 \mathrm{~s}$ & $7.76 \mathrm{~s}$ \\
\hline Fire & $7.91 \mathrm{~s}$ & $7.33 \mathrm{~s}$ & $12.58 \mathrm{~s}$ & $9.84 \mathrm{~s}$ & $6.96 \mathrm{~s}$ \\
\hline
\end{tabular}

With the following data gathered, the researchers analyzed these data to gain the needed information to attain the objectives of the research. The testing on the functionality of the device based on the different locations such as living room, terrace bedroom, dining area and kitchen in testing the time delay of the device and the time delay on sending SMS alert were conducted.

In Table 1 which is the testing of GF alert system on different location, it shows different time delay. At living room shows 0.75 second delay for the Gas Leakage detector to alert while 0.28 second on fire sensor. In terrace, it shows 1.19 seconds time delay on gas leakage detector while 0.97 second on fire sensor. On bedroom, it shows 0.49 second time delay on gas leakage and 0.15 second on fire sensor. In dining area, it shows 0.63 second time delay on gas leakage alert while 0.46 second time delay on fire sensor. In kitchen it shows 0.35 seconds time delay on gas leakage to detect while 0.22 second time delay on gas sensor.

Table 2 shows the delay on SMS alert, on living room shows 8.76 seconds time delay on using gas leakage while 7.91 seconds time delay using fire sensor. In terrace, it shows 6.93 seconds time delay using gas leakage sensor while
7.33 seconds time delay using fire sensor. On bedroom shows 12.09 seconds time delay using gas leakage sensor while 12.58 seconds time delay using fire sensor. In dining area, shows 10.15 seconds time delay using gas leakage sensor while 9.84 seconds time delay using fire sensor. In kitchen, shows 7.76 seconds time delay using gas leakage sensor while 6.96 seconds time delay using fire sensor.

Based on the analysis of the data on the table given it shows that the average time delay on living room is 0.515 second, 1.08 seconds on terrace, 0.32 second on bedroom, 0.545 second on dining area, and 0.285 second on kitchen. These data show that in kitchen has the shortest time delay while on terrace has longest time delay, therefore there are variables to be consider on placing the device.

On sending SMS alert it shows different time delay on different location. The average time delay for living room shows 8.335 seconds, 7.13 seconds on terrace, 12.335 seconds on bedroom, 9.995 seconds on dining area, and 7.36 seconds on kitchen. This show that on terrace with 7.13 seconds has the shortest time delay for the SMS to alert while on bedroom with 12.335 seconds has the longest time delay for the SMS to alert. 


\section{Effect of Place of Installation of the Device}

To determine the perfect location for installation the researchers conducted test and experimentation. Table 3 shows the time delay on sending SMS alert in different location.

Table 3. Testing of GF Alert in Sending SMS Alert with Corresponding Network Signal

\begin{tabular}{|l|l|l|l|l|l|}
\hline \multirow{2}{*}{} & Test 1 & Test 2 & Test 3 & Test 4 & Test 5 \\
\cline { 2 - 6 } & Living Room & Terrace & Bedroom & Dining Area & Kitchen \\
\hline Signal & 4 Bars & 5 Bars & 2 Bars & 3 Bars & 4 Bars \\
\hline Gas Leakage & $8.76 \mathrm{~s}$ & $6.93 \mathrm{~s}$ & $12.09 \mathrm{~s}$ & $10.15 \mathrm{~s}$ & $7.76 \mathrm{~s}$ \\
\hline Fire & $7.91 \mathrm{~s}$ & $7.33 \mathrm{~s}$ & $12.58 \mathrm{~s}$ & $9.84 \mathrm{~s}$ & $6.96 \mathrm{~s}$ \\
\hline
\end{tabular}

From the table, at the terrace which has 5 bars of signal transmitter there was 6.93 seconds time delay for gas leakage detector while 7.33 seconds time delay for the fire sensor to detect. On living room which has 4 bars of network signal shows 8.76 seconds time delay using gas leakage detector while 7.91 seconds time delay using fire sensor. On bedroom which has 2 bars of network signal shows 12.09 seconds time delay using gas leakage detector while 12.58 seconds time delay using fire sensor. On dining area which has 3 bars of network signal shows 10.15 seconds time delay using gas leakage detector while 9.84 seconds time delay using fire sensor. On kitchen which has 4 bars of network signal shows 7.76 seconds time delay using gas leakage detector while 6.96 seconds time delay using fire sensor.

In sending SMS alert the researcher analyzed the data to gather its average time delay and it shows at 4 bars the time delay in living room is 8.335 seconds, 7.13 seconds on terrace with 5 bars, 12.335 seconds on bedroom with 2 bars, 9.995 seconds on dining area with 3 bars, and 7.36 seconds on kitchen with 4 bars. This show that on terrace with 7.13 seconds has the shortest time delay for the SMS to alert while on bedroom with 12.335 seconds has the longest time delay for the SMS to alert. Therefore, the SMS alert is directly proportional to the Bars of network signal on specific location.

\section{CONCLUSIONS AND RECOMMENDATIONS \\ Conclusions}

Based on researchers' observation on testing of the device and evaluation of the findings, the following conclusion were drawn:

1.) The design of the system was unique and simple in construction, because the circuit only composed of integrated circuits. The researchers found difficulty on programming because it uses multiple sensor with GSM module.

2.) The system shows excellent response in detecting gas leaks and fire with short time delay. On the other hand, sending of SMS alert shows promising results with few seconds time delay.

3.) The network signal of the place of installation affects the performance of the device to send text messages.

\section{Recommendations}

From the conclusion mentioned above, the following recommendation were offered:

1.) Before installing the device, it must ensure that it has good network signal for better SMS alert. And also, the prepaid sim card must be kept with maintaining balance or use postpaid card for continuous service.

2.) Future researchers can upgrade the device by adding features such as power tripping device that will cut the supply when the gas sensor is triggered.

3.) Future researchers can also upgrade the system by adding sprinkler that will be triggered by the gas sensor or the fire sensor.

\section{REFERENCES}

1. Brushlinsky, N.N. Ahrens, M. Sokolov, S.V. and Wagner, P. (2016), "World fire statistics", CTIF, International Association of Fire and Rescue Services, No. 21, available at: www.ctif.org/sites/default/files/ctif_report21_world _fire_statistics_2016.pdf (accessed 30 June 2019).

2. https://www.usfa.fema.gov/downloads/pdf/statistic s/res_bldg_fire_estimates.pdf

3. http://erent.se/rise-of-qbr92/smoke-and-gasdetector-project.html

4. https://www.elprocus.com/gsm-technologyarchitecture-its-applications/Apeh, S. T. et al (2014). Design and Development of Kitchen Gas Leakage Detection and Automatic Gas Shut Off System

5. Abishek, V. et al (2013). Wireless Power Trip during Gas Leakage

6. Ismail, T. et al (2013). GSM Based Gas Leakage Warning System 
7. Georgewill, O. et al (2016). Design and Implementation of SMS-Based Industrial/Homes Gas Leakage Monitoring \& Detection Alarm System

8. Priya, D. H. et al (2014). Gas Leakage System

9. Shrivastava, A. et al (2013). GSM Based Gas Leakage Detection System

10. Ankit, S. et al (2015). Microcontroller Based LPG Gas Leakage Detector using GSM Module

11. Amsaveni, M. et al (2015). GSM Based LPG Leakage Detection and Controlling System

12. Liu, Z. et al (2008). Intelligent Residential Security Alarm and Remote-Control System Based on Single Chip Computer

13. Peijiang, C. et al (2008). Design and Implementation of Remote Monitoring System Based on GSM

14. www.circuitstoday.com GSM Based SMS Alert Fire Alarm System using Arduino

15. www.circuitstoday.com Gas Leakage Detector using Arduino and GSM Module with SMS Alert

16. www.edgefx.in Arduino LPG and Flame Detector 